\title{
Surrogate-Based Aerodynamic Optimisation of Compact Nacelle Aero-Engines
}

\author{
Fernando Tejero ${ }^{\mathrm{a}, *}$, David G MacManus ${ }^{\mathrm{a}}$, Christopher Sheaf ${ }^{\mathrm{b}}$ \\ ${ }^{a}$ Centre for Propulsion Engineering, School of Aerospace, Transport and Manufacturing, \\ Cranfield University, Bedfordshire, MK43 OAL \\ ${ }^{b}$ Rolls-Royce plc., P.O. box 31, Derby, United Kingdom, DE24 $8 B J$
}

\begin{abstract}
Genetic algorithms are a powerful optimisation technique for the design of complex engineering systems. Although computing power continuously grows, methods purely based on expensive numerical simulations are still challenging for the optimisation of aerodynamic components at an early stage of the design process. For this reason, response surface models are typically employed as a driver of the genetic algorithm. This reduces considerably the total overhead computational cost but at the expense of an inherent prediction uncertainty. Aero-engine nacelle design is a complex multi-objective optimisation problem due to the nonlinearity of transonic flow aerodynamics. This research develops a new framework, that combines surrogate modelling and numerical simulations, for the multiobjective optimisation of aero-engine nacelles. The method initially employs numerical simulations to guide the genetic algorithm through generations and uses a combination of higher fidelity results along with evolving surrogate models to identify a set of optimum designs. This new approach has been applied to the multi-objective optimisation of civil aero-engines which are representative of future turbofan configurations. Compared to the conventional CFD in-the-loop optimisation method, the proposed algorithm successfully identified the same set of optimum nacelle designs at a $25 \%$ reduction in the computational cost. Within the context of preliminary design, the method meets the typical $5 \%$
\end{abstract}


acceptability criterion with a $65 \%$ reduction in computational cost.

Keywords: Optimisation, surrogate model, Nacelle, aerodynamics, aero-engine.

\section{Nomenclature}

\section{Roman Symbols}

R Correlation matrix

A Area

$C_{D \text {-cruise }}$ Nacelle drag evaluated at cruise point

$C_{D-\text { spill }}$ Nacelle drag evaluated between end of cruise and mid cruise

$D \quad$ Drag

$f \quad$ Non-dimensional factor

$h \quad$ Altitude

$L \quad$ Length

$M \quad$ Mach Number

$N_{\text {Pearson }}$ Pearson's product moment of correlation

$P \quad$ Pressure

$R \quad$ Correlation function

$r \quad$ Radius

${ }^{*}$ Corresponding author

E-mail address: f.tejero@cranfield.ac.uk 


$\begin{array}{ll}V & \text { Velocity } \\ x & \text { Abscissa } \\ Z & \text { Stochastic process }\end{array}$

\section{Greek Symbols}

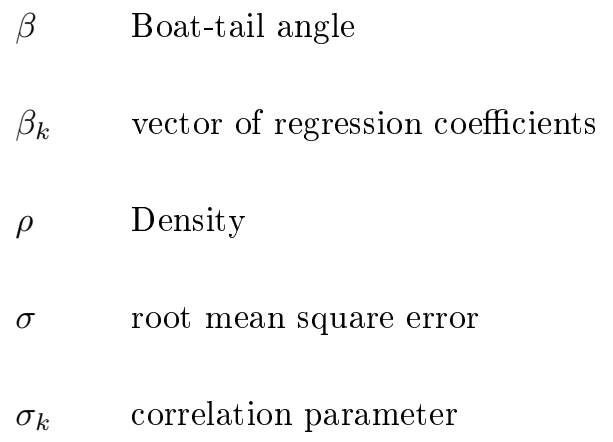

\section{Superscripts and Subscripts}

$\begin{array}{ll}\infty & \text { Freestream } \\ D R & \text { Drag Rise } \\ h i & \text { Highlight } \\ \text { if } & \text { Initial Forebody } \\ \text { max } & \text { Maximum radius } \\ n a c & \text { Nacelle } \\ T E & \text { Trailing Edge }\end{array}$

Acronyms

AM Adaptive Method
CFD Computational Fluid Dynamics 
CST Class Shape Transformation

DSE Design Space Exploration

GA Genetic Algorithm

GPR Gaussian Process Regression

LHS Latin Hypercube Sampling

MFCR Mass Flow Capture Ratio

MOO Multi-objective optimisation

PF Pareto Front

RANS Reynolds Averaged Navier Stokes

RSM Response Surface Model

SST Shear Stress Transport

TM Traditional Method

\section{Introduction}

Aerodynamic shape optimisation has become an indispensable method to meet the recent aerospace performance targets established by the Advisory Council for Aviation Research and Innovation in Europe (ACARE) in Flightpath 2050 [1], and by NASA [2]. For example, relative to year-2000 aircraft, Flightpath 2050 aims for reductions of $75 \%$ in fuel-burn, $65 \%$ in perceived noise, and $80 \%$ in landing/take-off $N O_{X}$ emissions. To achieve these challenges, novel and innovative frameworks for the optimisation of multidimensional aerodynamic problems, which include numerical simulations, surrogate modelling, advance shape parametrisation and adequate optimisation routines, are being developed 
[3]. Concerning aero-engine nacelle design, it is envisaged that future aeroengines will operate at higher bypass ratio (BPR) [4] and lower fan pressure ratio (FPR) [5] to reduce the engine specific fuel consumption (SFC) and to improve its propulsive efficiency. These new architectures will have larger engine diameters, which results in an increase of the nacelle weight, overall aircraft drag and interaction effects with the airframe [6]. Thus, there is a need to develop methods for the design of compact nacelles that do not counteract the efficiency gained from the reduction of the specific thrust [7].

Although computing power continuously grows, methods based on expensive numerical simulations are still not always suitable for complex aerodynamic optimisation due to the associated large computational overhead cost. For this reason, the popularity of response surface models (RSM) has grown in recent years $[8,9]$. Nevertheless, there are still many engineering applications for which the non-linear characteristics present particular difficulties for low-order models. Several methods have been used to model transonic flow aerodynamics such as polynomial response surfaces [10], radial basis functions [11] or neural networks [12]. For aircraft optimisation applications, the Kriging method [13] has been demonstrated to be useful for complex design spaces [14, 15, 16].

Within an optimisation framework, Genetic Algorithms (GAs) are known to be suitable for complex multi-objective optimisation (MOO) problems due to its global convergence characteristics [17]. A key aspect is to enable a tractable investigation and efficient coverage of the design space. In this respect, a robust and sufficiently flexible parametric representation of the aero-geometry is required to carry out the associated shape manipulations. A comprehensive review of different parameterizations for aerodynamic shapes was carried out by Masters et al. [18], who concluded that the Class Shape Transformation (CST) method had an acceptable coverage of the investigated aerofoil design space. 


\subsection{Aerodynamic Nacelle design}

Nacelle design is a multi-disciplinary process in which geometrical constraints and aerodynamic requirements need to be fulfilled [19]. Designers usually set the nacelle length $\left(L_{n a c}\right)$ and maximum nacelle radius $\left(r_{\max }\right)$ (Figure 1) to ensure space for other engine sub-systems such as the thrust reverser. Besides, the nacelle trailing edge location $\left(r_{t e}\right)$ is selected to meet the engine nozzle requirements and to reduce adverse effects due to the installation on to the airframe. Nacelles need to perform adequately across the range of operating conditions that are met during the aircraft mission. This highlights the suitability of nacelle design by multi-objective optimisation (MOO). Within the cruise segment, one of the main performance metrics of interest is the nacelle drag at mid-cruise conditions $\left(C_{D-\text {-cruise }}\right)$, which is minimized to reduce the overall fuel consumption. The value at which the nacelle drag, as a function of freestream Mach number $\left(M_{\infty}\right)$ at a constant mass-flow capture ratio $(M F C R)$, increases sharply is defined as drag rise Mach number $\left(M_{D R}\right)$. This performance metric is typically maximised and to provide operational flexibility a margin of 0.02 between cruise Mach number and drag rise Mach number is targeted to ensure that an increase of $M_{\infty}$ does not have an adverse impact on the nacelle performance [20]. Lastly, the variation in nacelle drag as the mass flow capture ratio reduces during the aircraft cruise mission is defined as spillage drag $\left(C_{D-\text { spill }}\right)[21]$ and is also minimized.

There are very few investigations in the open literature concerning nacelle design by means of surrogate modelling. Robinson et al. [23] developed a Response Surface Model (RSM) using a Gaussian Process Regression (Kriging interpolation) as a driver for the GA. The influence of the initial sample, as well as the number of individuals for the subsequent generations, was investigated. The potential benefit from using a larger initial generation and reduced overall 


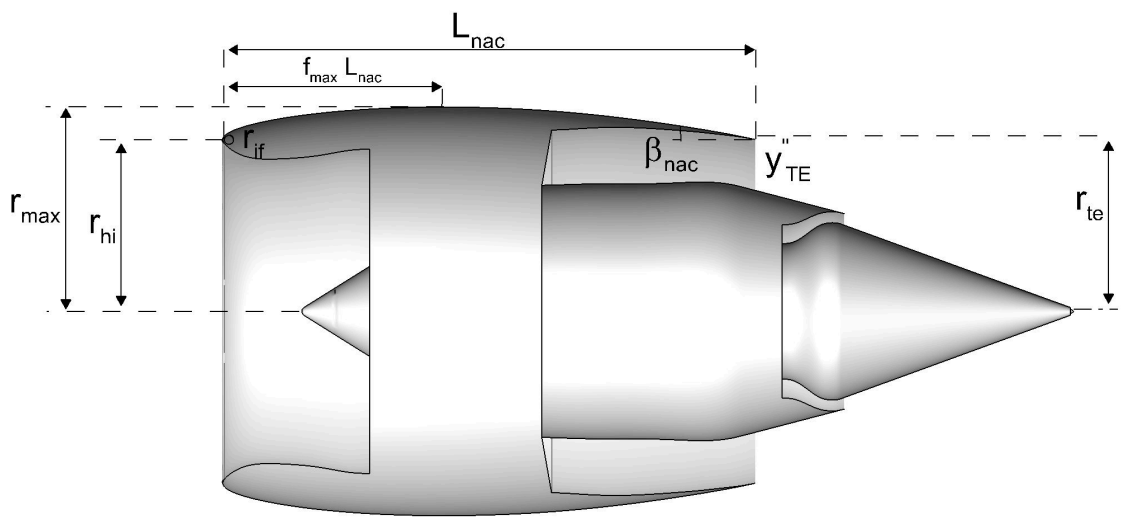

Figure 1: Nacelle geometry parametrisation (based on [22])

generations was demonstrated. Song and Keane [16] carried out the shape optimisation of a civil aircraft engine nacelle in transonic conditions $\left(M_{\infty}=0.85\right)$. A Kriging-based surrogate model containing 33 design variables was employed to drive the genetic algorithm to a set of optimum nacelle designs. The Kriging model was updated using the CFD results of the Pareto front points and the process was looped until the solution converged or the computational budget was exhausted. The authors reported the difficulties to obtain a converged Pareto front due to the Kriging model accuracy. A hybrid genetic algorithm that combined a Kriging surrogate model and RANS numerical simulations for the aerodynamic optimisation of a transonic nacelle $\left(M_{\infty}=0.80\right)$ was carried out by Fang et al. [24]. The initial design space exploration and subsequent generations were formed by 32 nacelle designs in which about $5 \%$ of the individuals were evaluated with the Kriging response method. The accuracy of the metamodel was improved by updating the model with additional CFD data through generations. This strategy was applied to optimise the cruise drag $\left(C_{D-\text { cruise }}\right)$ of an axisymmetric short nacelle $\left(L_{n a c} / r_{h i}=3.32\right)$ by 1.5 aircraft drag counts with respect to a baseline design. Sasaki et al. [25] performed the aerodynamic optimisation of a nacelle for an over-wing mounted engine. Within this study, 
the nacelle position, nacelle shape and wing shape were optimised using a hybrid genetic algorithm that combined a Kriging model and Euler CFD solutions. The investigation was based on the DLR-F6 airframe at a flight Mach number of $M_{\infty}=0.70$. Relative to a baseline configuration with aircraft $L / D=25.0$, an optimum design with an improvement of $38 \%$ was found $(L / D=34.5)$. Zhong and $\operatorname{Li}[26,27]$ also used a hybrid approach which combined a Kriging surface model and RANS CFD simulations to maximize the delay of boundary layer transition for a nacelle at transonic conditions $\left(M_{\infty}=0.78\right)$. Following this strategy, a new nacelle design with $7 \%$ larger area of laminar flow with respect to a baseline case was designed.

\subsection{Scope of the present work}

Within the aforementioned context, there is a clear need to employ low order models for the multi-objective optimisation of compact nacelle aero-engines at an early stage of the design process. This paper aims to develop a new method based on a Design Space Exploration (DSE) combined with an adaptive surrogate model for the multi-objective optimisation of compact nacelles for future aero-engines. The tool includes a set of geometry definitions using intuitive Class Shape Transformations (CST) $[28,29]$ for the representation of the intake, nacelle and exhaust, a multi-block structured automatic mesh generation tool [30], computation of the viscous compressible flow-field [31], extraction of the nacelle performance metrics of interest, surrogate modeling [13] and a genetic algorithm procedure [17].

A thorough investigation of meta-models predictive accuracy within an optimisation environment for the complex nonlinear transonic flow aerodynamics associated to nacelles is not available in the open literature. For this work two different strategies are considered to structure response surface models: (a) traditional design space exploration and (b) developed adaptive method. The 
influence of the RSM sample size in the prediction uncertainty has been quantified. Besides, the results are used to derive guidelines for the multi-objective optimisation of civil aero-engines with meta-models.

\section{Methods and scope}

The present work expands the capability of the computational framework developed by Tejero et al. [22] for the multi-objective optimisation of nacelle aero-lines within the new design space for future aero-engines. The framework was previously demonstrated to capture nacelle design sensitivities for different nacelle length, trailing edge radii as well as operating conditions. Due to the inherent non-linearity of transonic flow aerodynamics associated with aeroengines, there was a noticeable computational cost associated with the large number of CFD simulations required to find a set of optimal solutions. For example, the MOO optimisation routine was typically carried out with the established method of an initial design space exploration of 400 samples and 30 subsequent generations of 50 evaluations each [23, 22], in which every nacelle design was evaluated by means of numerical simulations for a range of aerodynamic operating conditions.

Within this work, the framework has been extended to accommodate the capabilities for a more computationally efficient optimisation strategy based on Design of Experiments (DOE), Response Surface Model (RSM) and a Genetic Algorithm (GA). Multi-objective optimisation based on RSMs is a wellestablished method [15, 32, 33]. Usually, the optimisation strategy is initialized with a comprehensive design space exploration based on an isotropic sampling such as the Latin Hypercube Sampling (LHS) [34, 35]. Then, a surrogate model is built from the DOE and used to drive the GA during the optimisation process. This method is referred in this work as traditional method or TM. The 
approach has been employed successfully for the optimisation of nozzle exhaust aerodynamic shapes [15], rotorcraft missions [32] and aircraft wings [33]. Nevertheless, its application to the non-linear multi-objective optimisation problem of nacelle design is limited due to the inherent difficulties to build an accurate response surface model.

This current work develops a MOO strategy based on adaptive surrogate modelling, referred in the next sections as adaptive method or AM. The framework employes CFD simulations to carry out the design space exploration as well as to evaluate the nacelle designs in a fixed number of subsequent generations. Then, all CFD data is used to generate a RSM to guide the GA until reaching convergence to the Pareto front. Following this approach, the GA is guided to the regions of the design space where optimum designs are located because the first generations are carried out by means of numerical simulations. In addition, the surrogate model has more CFD data in the areas of interest which increases the meta-model accuracy and, therefore, of the predicted Pareto front.

\subsection{Nacelle parametric design}

A robust and flexible parametric representation of the nacelle aero-lines is required to investigate the aerodynamic performance of compact nacelles. The method should be suitable to cover efficiently all the design space, while keeping the number of design variables to a minimum. Within the context of this work, this is an important aspect for the present DOE approach coupled with a surrogate model and genetic algorithm due to the inherent difficulties to fit accurate structured RSM models in high dimensional problems [16].

The developed framework incorporates a parametric geometry definition of the main aero-engine installation components, such as nacelle, intake and exhaust system, based on Class-Shape Transformations (CST) [28]. The original 
method described by Kulfan has been adapted to employ intuitive design variables as presented by Christie et al. [29]. Although nacelles are designed as a combination of axisymmetric aero-lines to define a 3D geometry, the key step in the design process lies in the design of each aero-line. For the present work, eight design variables are employed in the parametric geometry definition of a

single nacelle aero-line, i.e. $r_{t e}, r_{h i}, L_{n a c}, f_{\max }, r_{\max }, r_{i f}, \beta_{\text {nac }}$ and $y_{t e}^{\prime \prime}$ (Figure 1) and a detailed description of the fan cowl parametrisation has been reported by Tejero et al. [22].

\subsection{Computational method}

The compressible steady Favre-averaged Navier-Stokes equations were solved with a compressible, implicit, density-based solver [31]. A second-order accurate upwind spatial discretization with the Green-Gauss node based scheme was used for the computation of the flow-field gradients. Sutherland's law was employed for the calculation of the dynamic viscosity. The CFD simulations were carried out with the $k-\omega$ SST turbulence closure [36].

The freestream conditions were specified using a pressure far-field boundary condition by setting static pressure $P_{s t}$, static temperature $T_{s t}$ and Mach number $M_{\infty}$. The computational domain includes the engine intake to account for the effect of mass flow capture ratio (MFCR) on the nacelle pressure distribution which is controlled by a pressure-outlet and fixes the mass-flow through the engine. The inlet conditions to the bypass and core nozzle ducts were prescribed using $P_{t}$ and $T_{t}$. To minimise exhaust interference on the nacelle drag the $P_{t}$ and $T_{t}$ was set to provide a nominally isentropic expansion.

Previous work established the mesh independence requirements for the design of axisymmetric nacelle aerolines [37]. For the multi-block structured mesh a grid convergence index (GCI) [38] of $1 \%$ for nacelle drag was determined based on meshes of 10,000, 20,000 and 40,000 cells. Therefore, the current work used 


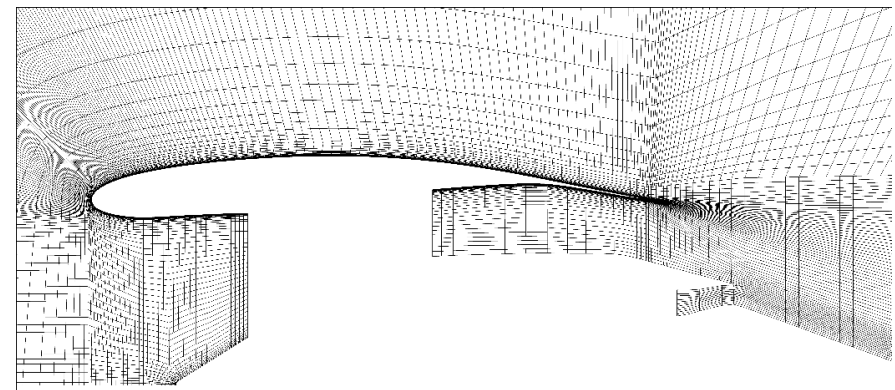

Figure 2: Mesh close-up near the engine surfaces

the 20,000 cells mesh. A domain sensitivity study for four different domain sizes $\left(40 r_{\max }, 60 r_{\max }, 80 r_{\max }, 100 r_{\max }\right)$ demonstrated that the changes on $C_{D-\text { cruise }}$ were on the order of $0.1 \%$ for the chosen $80 r_{\max }$ domain size. Figure 2 presents a close-up near the engine surfaces for the mesh employed throughout this study. The accuracy of the numerical approach was tested against publicly available configurations [39]. The experimental data collected a wide range of aerodynamic points, changing the Mach number from 0.65 to 0.95 and MFCR from 0.40 to 0.60 . For this range of operating conditions, the predicted nacelle drag was typically within $3.5 \%$ of the experimental data and the CFD accuracy for drag rise divergence $\left(M_{D R}\right)$ was within 0.005 [37].

\subsection{Surrogate modelling}

Within this work, each nacelle design analysed by means of numerical simulation was used as input to build the surrogate models. An interpolation model was employed with a Gaussian Process Regression (GPR) based on the ordinary Kriging interpolation method [13] with a quadratic regression function and an absolute exponential auto-correlation. The Kriging model approximates a function, $y(x)$, that can be expressed as a linear combination of $f(x)$, a polynomial approximation of $x$, and a stochastic process with zero mean and standard deviation, $Z(x)($ Eq. 1) [13]: 


$$
y(x)=f(x)+Z(x)
$$

For the purpose of this work, $f(x)$ is defined as a linear combination of polynomials of second order, $f_{k}(x)$, i.e. quadratic regression function, as defined in Eq. 2.

$$
f(x)=\sum_{k=1}^{p} \beta_{k} f_{k}(x)
$$

where $\beta$ is the $\mathrm{p}$-dimensional vector of regression coefficients.

On the other hand, while $f(x)$ globally approximates the design space, the stochastic process, $Z(x)$, creates localized deviations so that the Kriging model interpolates the $N_{s}$ sampled data points [40]. The covariance matrix of $Z(x)$ is defined by Eq. 3 [40]:

$$
\operatorname{Cov}\left[Z\left(x^{i}\right), Z\left(x^{j}\right)\right]=\sigma^{2} \mathbf{R}\left(R\left(x^{i}, x^{j}\right)\right)
$$

where $\mathbf{R}$ is the correlation matrix and $R\left(x^{i}, x^{j}\right)$ is the correlation function between the $i$ and $j$ points of the $N_{s}$ sampled data points. For this work, $R\left(x^{i}, x^{j}\right)$ is defined as the Gaussian correlation function given by Eq. 4 [40]:

$$
R\left(x^{i}, x^{j}\right)=\exp \left[\sum_{k=1}^{n_{d}} \theta_{k}\left|x_{k}^{i}-x_{k}^{j}\right|^{2}\right]
$$

where $n_{d}$ refers to the number of design variables, $\theta_{k}$ is an unknown correlation parameter that needs to be calculated to be fitted in the model and $x_{k}^{i}$ and $x_{k}^{j}$ are the $k$-th component of the $x^{i}$ and $x^{j}$ sample points [40].

The Kriging predictions, $\hat{y}$, which are estimations of the response surface model $y(x)$, are given by the Eq. 5: 


$$
\hat{y}=\hat{\beta}+r^{T}(x) \mathbf{R}^{-1}(y-f \hat{\beta})
$$

where $y$ is the column vector of length $N_{s}$ that contains the sample values of the response, $f$ is the unity column vector of length $N_{s}$ when $f(x)$ is taken as a constant, and $r^{T}(x)$ is a vector in function of the correlation function of the $N_{s}$ data samples.

The unknown variable $\hat{\beta}$ in Eq. 5 can be estimated by Eq. 6

$$
\hat{\beta}=\left(f^{T} R^{-1} f\right)^{-1} f^{T} R^{-1} y
$$

To close this system of non-linear equations, the unknown parameter $\theta_{k}$ is calculated by maximizing the Eq. 7 :

$$
\max \left(\theta_{k}\right)=-\left[N \ln \left(\hat{\sigma}^{2}+\ln |\mathbf{R}|\right] / 2\right.
$$

The Leave-One-Out (LOO) cross-validation [41] was employed to assess the predictive accuracy of the structured RSMs. It is a $k$-fold method in which $k$ is equal to the number of data samples. Within the context of this work, the response surface models are built with a Gaussian process regression based on the Kriging interpolation method. Although the model provides the mean value of the Gaussian distribution as well as the standard deviation, only the mean value is considered for the nacelle drag prediction throughout this investigation. Therefore, the procedure for the LOO cross-validation and the nacelle drag predictions are equivalent. Although the Gaussian Process probabilistically takes into account the uncertainty of the model, its accuracy was not assessed with a Bayesian-based method [42] because the uncertainty is not considered during the optimisation process. During the present LOO cross-validation, the obtained surrogate model predictions are correlated against the respective data 
points in terms of Pearson's product moment of correlation [43] along with the gradient of the associated linear regression line to assess the predictive accuracy of the response surface model.

\subsection{Genetic Algorithm}

The non-dominated sorting genetic algorithm (NSGA-II), proposed originally by Deb [17], has been used in this work due to its proven capabilities for global optimisitation in flow aerodynamic applications [22]. The investigation was carried out with a Gaussian mutation operation and a mutation rate of 0.2 , which is inversely proportional to the number of nacelle design variables as suggested by Deb [44]. Previous investigations for compact nacelle design optimisation have demonstrated the sensitivity of the mutation standard deviation size $\left(\sigma_{m}\right)$ on the Pareto front convergence $[45,23]$. This study concluded that $\sigma_{m}=0.0005$ ensured the best compromise for a fast convergence to the Pareto front solution at a reduced risk of being trapped in a local optima. Therefore, the same value has been used throughout this investigation. The crossover used the BLX- $\alpha$ operator with $\alpha=0.5$, as suggested by Eshelman and Schaffer [46], which results in an even split between the two parent designs and has been proven to outperform other values of $\alpha$ in a wide range of benchmark functions [46]. The initial seed was set to 400 , with 50 individuals in following generations and a total number of 30 generations. The selection of these parameters for nacelle multi-objective optimisation is based on a statistical investigation where the initial seed and sample size was investigated in which it was concluded that the selected settings improve the convergence to the Pareto optimal set [23]. The convergence of the Pareto front solution was assessed by calculating the hypervolume through generations [47]. For the current investigation, the changes in the hypervolume was below $1 \%$ for the last 3 generations, which ensured the convergence to the Pareto front. 
Three nacelle performance metrics were employed as objective functions for the present optimisation routine. The nacelle cruise drag $\left(C_{D-\text { cruise }}\right.$, Eq. 8$)$ was calculated following the drag extraction method described by Christie et al. [29]. The sensitivity of the nacelle to Mach number was evaluated by the change in nacelle drag $\left(\Delta C_{D-M a c h}\right.$, Eq. 9$)$ at cruise Mach number $\left(M_{r e f}=M_{\infty}\right)$ and an increased conditions of $M_{\text {delta }}=M_{\text {ref }}+0.02$. The third performance metric was the spillage drag $\left(C_{D-\text { spill }}\right.$, Eq. 10$)$ which is defined as the change of nacelle drag due to the change in MFCR between a typical end-of-cruise and mid-cruise conditions.

$$
\begin{gathered}
C_{D-\text { cruise }}=\frac{D_{\text {nac }}}{\frac{1}{2} \rho_{\infty} V_{\text {ref }}^{2} A_{h i}} \\
\Delta C_{D-M a c h}=\frac{D_{n a c, M=M_{\text {delta }}-D_{n a c, M=M_{\text {ref }}}}}{\frac{1}{2} \rho_{\infty} V_{r e f}^{2} A_{h i}} \\
C_{D-\text { spill }}=\frac{D_{\text {nac }, M F C R_{\text {cruise }}-D_{n a c, M F C R_{E O C}}}}{\frac{1}{2} \rho_{\infty} V_{r e f}^{2} A_{h i}}
\end{gathered}
$$

\section{Results and analysis}

The design of compact nacelles for future aero-engines is a well-posed problem for multi-objective optimisation due to the range of operating conditions that are met throughout the aircraft mission. This section describes three different strategies for the identification of the Pareto front (a) purely based on numerical simulations, (b) surrogate model constructed with a single design space exploration and (c) the proposed adaptive hybrid response surface modeling approach. To investigate the applicability of each MOO strategy, a compact civil aero-engine with $L_{n a c} / r_{h i}=3.0$ and $r_{t e} / r_{h i}=1.0$ has been selected. This compact nacelle configuration is not in a conventional part of the design space 
and previous work [22] highlighted the non-linear characteristics of the design space associated with the transonic flow-field. In that context, it is a challenging design to test the proposed new MOO approach. The first MOO strategy, i.e. CFD in-the-loop, is employed as the baseline of the optimisation process and the Pareto front is used for comparison purposes with the other algorithms. The influence of the initial DOE sampling on the Pareto front prediction has been investigated with the method based on a design space exploration. Five independent LHS were carried out with a total computational overhead cost of $20 \%, 35 \%, 50 \%, 62 \%$ and $75 \%$ with respect to the CFD in-the-loop method (TM in Table 1). Lastly, four different models with the proposed adaptive hybrid response surface modeling approach were developed with an overall computational cost of $35 \%, 50 \%, 62 \%$ and $75 \%$ with respect to the CFD in-the-loop strategy (AM in Table 1).

For this study, the operating conditions at mid-cruise were set to a flight Mach number of $M_{\infty}=0.85$, MFCR $=0.70$ and $\mathrm{h}=10668 \mathrm{~m}$, which is representative of expected conditions for future aero-engines. The end-of-cruise was set to $M_{\infty}=0.85, \mathrm{MFCR}=0.65$ and $\mathrm{h}=10668 \mathrm{~m}$. During the multi-objective optimisation process, the design variables $r_{t e}, r_{h i}$ and $L_{n a c}$ are constant while the remaining five parametric design variables $\left(f_{\max }, r_{\max }, r_{i f}, \beta_{n a c}\right.$ and $\left.y_{t e}^{\prime \prime}\right)$ are variable. The lower and upper bound of these five parameters were set to avoid non-monotic curvature distributions on the nacelle geometries (Table 2).

\subsection{Nacelle Multi-Objective Optimisation based on the full CFD in-the-loop method}

The non-linearity of the nacelle aerodynamic design of this engine configuration is highlighted in the three-dimensional Pareto surface of Figure 3a. This Pareto front has been obtained with the conventional method of the CFD in-theloop process that requires large computational resources but provides a higher 
Table 1: Summary of the cases considered for the nacelle aero-line multi-objective optimisation

\begin{tabular}{ccccccc}
$\begin{array}{c}\text { Method for } \\
\text { MOO }\end{array}$ & $\begin{array}{c}\text { LHS seed } \\
N_{\text {seed }}\end{array}$ & $\begin{array}{c}\text { CFD gen. } \\
N_{\text {gen }}\end{array}$ & $\begin{array}{c}\text { CFD des./gen. } \\
N_{s}\end{array}$ & $\begin{array}{c}\text { RSM gen. } \\
N_{\text {gen }-R S M}\end{array}$ & $\begin{array}{c}\text { RSM des./gen. } \\
N_{s-R S M}\end{array}$ & Total CFD cost \\
\hline CFD & 400 & 30 & 50 & - & - & 5550 \\
\hline TM1 & 400 & - & - & 30 & 50 & 1200 \\
TM2 & 650 & - & - & 30 & 50 & 1950 \\
TM3 & 900 & - & - & 30 & 50 & 2700 \\
TM4 & 1150 & - & - & 30 & 50 & 3450 \\
TM5 & 1400 & - & - & 30 & 50 & 4200 \\
\hline AM2 & 400 & 5 & 50 & 30 & 50 & 1950 \\
AM3 & 400 & 10 & 50 & 30 & 50 & 2700 \\
AM4 & 400 & 15 & 50 & 30 & 50 & 3450 \\
AM5 & 400 & 20 & 50 & 30 & 50 & 4200 \\
\hline
\end{tabular}

Table 2: Design variable bounds for the MOO of compact nacelles

\begin{tabular}{lcc}
\hline Parameter & Upper bound & Lower bound \\
\hline$f_{\max }[-]$ & 0.25 & 0.5 \\
$r_{\max } / r_{h i}[-]$ & 1.1 & 1.3 \\
$f_{i f}[-]$ & 0.6 & 1.4 \\
$y_{T E}^{\prime \prime} / r_{h i}[-]$ & -0.1 & 0.0 \\
$\beta_{\text {nac }}\left[^{\circ}\right]$ & 11 & 14 \\
\hline
\end{tabular}

fidelity on the nacelle drag estimation than other conventional reduced order methods. The projection of the Pareto surface is represented in the $C_{D-c r u i s e}$ - $\Delta C_{D-M a c h}$ space, and colored by $C_{D-s p i l l}$, where the dominated and nondominated solutions are marked with "+" and "o", respectively. Through the optimisation process the nacelle cruise drag was reduced by $4.7 \%$, varying from $C_{D-\text { cruise }}=0.0300$ for the best nacelle identified in the initial design of experiments to $C_{D-\text { cruise }}=0.0284$ at the end of the optimisation process. The Pareto surface clearly highlights some of the classical aspects of transonic nacelle design with trade-offs between cruise drag and off-design performance in terms of sensitivity to Mach number and spillage.

For example, the performance metrics for the individuals with the lowest 
$C_{D-\text { cruise }}, \Delta C_{D-\text { Mach }}$ and $C_{D-\text { spill }}$ (A1, A2, A3, respectively in Figure 3a) are summarized in the Table 3 . Relative to A1, which has the lowest cruise drag $\left(C_{D-\text { cruise }}=0.0284\right), \mathrm{A} 2$ has $\Delta C_{D-\text { Mach }} \approx 0$ but at the expense of a $22 \%$ increase in $C_{D-\text { cruise }}$. On the other hand, A3 can achieve effectively no spillage drag $\left(C_{D-\text { spill }} \approx 0\right)$ but with a $6.7 \%$ increase in cruise drag and a large concomitant $\Delta C_{D-M a c h}=0.0182$.

The main advantage of this MOO approach, based on higher fidelity numerical simulations, is that the integral values of the nacelle drag characteristics, i.e. $C_{D-\text { cruise }}, \Delta C_{D-M a c h}, C_{D-\text { spill }}$, as well as the lift distribution along the fan cowl are available within the framework without any extra computational effort. For instance, Figure 3b presents the isentropic Mach number distributions at cruise conditions for the nacelle designs $\mathrm{A} 1, \mathrm{~A} 2$ and $\mathrm{A} 3$. While $\mathrm{A} 1$ presents the largest peak Mach number $M_{i s}=1.35$, the A2 and A3 designs exhibit a lower acceleration over the lip with a maximum $M_{i s}$ of 1.28 and 1.21 , respectively. Downstream of the peak suction location there are also noticeable differences in the lift distributions for these designs (Figure 3b). A1 has a smooth and monotonic reduction of $M_{i s}$ with a weak shock wave at $x / L_{n a c}=0.37$ and the designs A2 and A3 present a well defined and strong shock topology. The nacelle A2, which has the lowest $\Delta C_{D-M a c h}$, presents a double shock wave that has associated large shock losses and results in a large $C_{D-\text { cruise }}=0.0345$. This nacelle has a first pre-shock $M_{i s}$ of 1.17 at $x / L_{n a c}=0.31$, located upstream of the maximum nacelle thickness. Then, the flow accelerates along the nacelle afterbody resulting in a second strong shock with pre-shock $M_{i s}$ of 1.23 at $x / L_{n a c}=0.66$. The sample A2 is not considered as a likely design as the cruise drag penalty is too large (Table 3 ) and the $M_{i s}$ distribution too severe (Figure $3 \mathrm{~b})$. On the other hand, the A3 design, which is the least sensitive to MFCR with a $C_{D-\text { spill }} \approx 0$, presents a well defined normal shock with a pre-shock $M_{i s}$ 
Table 3: Performance metrics for selected nacelle designs

\begin{tabular}{lccc}
\hline & $C_{D \text {-cruise }}$ & $\Delta C_{D-\text { Mach }}$ & $C_{D-\text { spill }}$ \\
\hline Nacelle A1 & 0.0284 & 0.0059 & 0.0016 \\
Nacelle A2 & 0.0345 & $\approx 0$ & 0.0006 \\
Nacelle A3 & 0.0303 & 0.0182 & $\approx 0$ \\
\hline
\end{tabular}

of 1.14 at $x / L_{n a c}=0.57$. All three configurations present notable differences on the nacelle aero-line geometries (Figure 3c). While the sample A1 is the most compact design, A2 presents the largest $r_{\max } / r_{h i}$. For the selected designs, the forebody length ratio varies from $f_{\max }=0.38$ to 0.44 and the boat-tail angle has a similar value. The initial forebody radius $\left(r_{i f}\right)$ reduces with $r_{\max } / r_{h i}$ in order to meet the nacelle end points. It increases the external lip curvature which results in a larger acceleration over the nacelle lip. As such, the most compact nacelle A1 present the largest peak Mach number $M_{i s}=1.35$.

This case of MOO for transonic nacelle design demonstrates that the CFD in-the-loop optimisation can be successfully used to identify the Pareto surface of a compact aero-engine configuration. This example required 30 optimisation generations and a total of 1850 nacelle design evaluations by means of CFD for the optimisation of a single axisymmetric nacelle aero-line with specific $L_{n a c} / r_{h i}$ $=3.0$ and $r_{t e} / r_{h i}=1.0$. Overall, 5550 CFD simulations were carried out during the optimisation routine to evaluate each nacelle design at cruise conditions $\left(C_{D-\text { cruise }}\right)$ and at two off-design conditions $\left(\Delta C_{D-\text { Mach }}\right.$ and $\left.C_{D-\text { spill }}\right)$. Consequently there is a desire to reduce the computational effort, while retaining the optimisation performance.

3.2. Nacelle Multi-Objective Optimisation by surrogate modelling based on the

\section{LHS DOE method}

The framework has been adapted to use a more computationally efficient strategy for nacelle aero-line MOO based on surrogate modelling. This is a rel- 


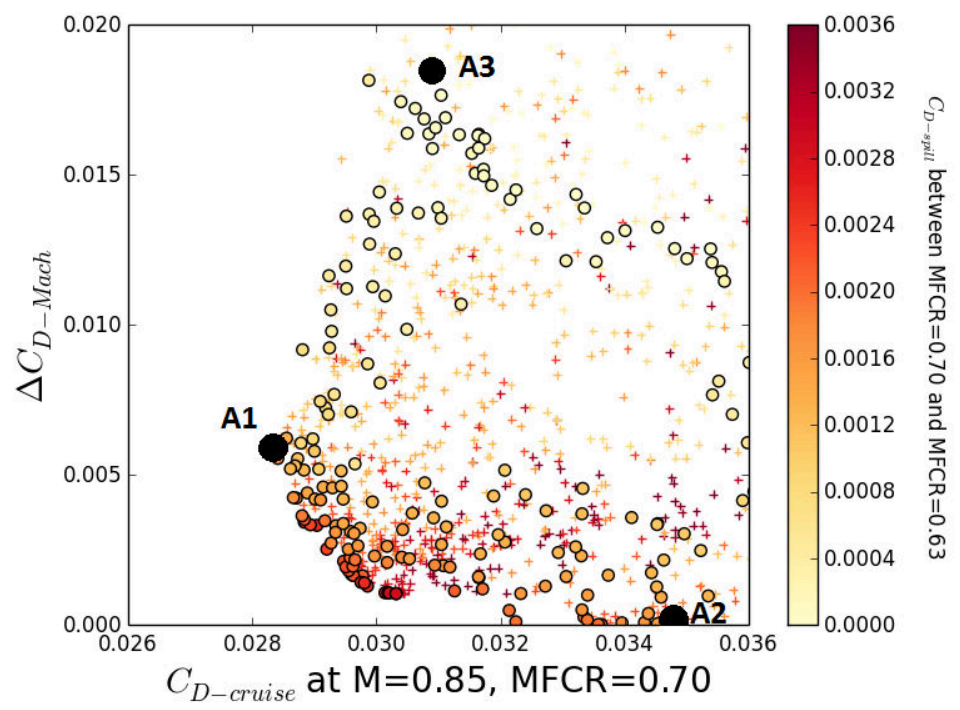

(a) Pareto front

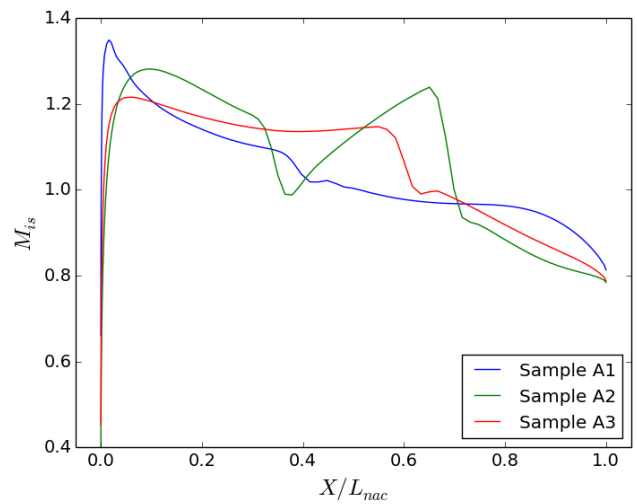

(b) Lift distribution at cruise conditions

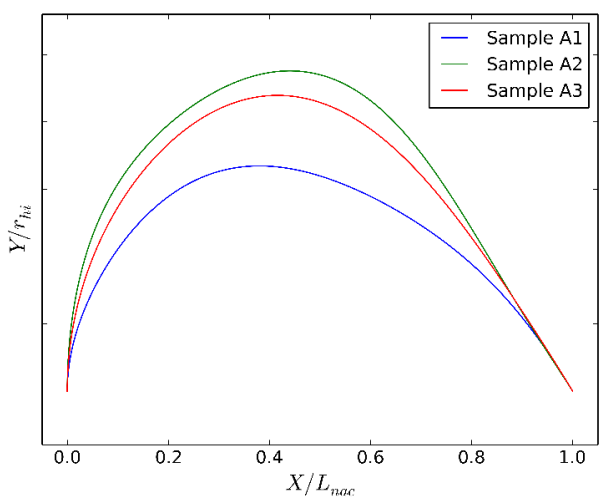

(c) Geometry comparison

Figure 3: (a) Pareto front obtained with the MQO CFD in-the-loop approach for the compact nacelle with $L_{n a c} / r_{h i}=3.0$ and $r_{t e} / r_{h i}=1.0$, (b) lift distribution comparison and (c) nacelle geometry comparison 
atively conventional approach in which a design space exploration is carried out with an isotropic Latin Hypercube Sampling (LHS) [34, 35]. All the samples are employed to generate a meta-model for the prediction of the nacelle drag, which then drives the GA to a set of optimum designs. This approach reduces the considerable overhead cost of the multiple CFD evaluations required throughout the optimisation process. A thorough investigation of the influence of DOE sampling size on the Pareto front prediction is assessed in this section.

Five independent LHS DOEs have been carried out with a total number of $400,650,900,1150$ and 1400 nacelle designs, referred in the text as TM1, TM2, TM3, TM4 and TM5, respectively (Table 1). Therefore, relative to the conventional CFD in-the-loop method, the computational overhead cost of each LHS DOE model is between $20 \%$ and $75 \%$ (Table 1). The performance metrics for each design were calculated by means of numerical simulations and the results were used to create five different RSMs using a Gaussian Process Regression (GPR) based on a Kriging interpolation method [13]. For each independent surrogate model, the Leave-One-Out (LOO) cross-validation [41] was employed to assess the predictive accuracy of the structured RSMs in the three nacelle performance metrics of interest, i.e. $C_{D-\text { cruise }}, \Delta C_{D-M a c h}, C_{D-\text { spill }}$ (Figure 4). Based on the linear correlation coefficient $\left(N_{\text {Pearson }}\right)$ [43], the computed quality of the surrogate model with 400 data samples (TM1) was $0.956,0.925$ and 0.977 for $C_{D-\text { cruise }}, \Delta C_{D-M a c h}, C_{D-\text { spill }}$, respectively. The root mean square error of this model $\left(\sigma_{L H S}\right)$, which is based on a Latin hypercube sampling, was $14.1 \%$, $11.3 \%$ and $13.3 \%$ for the three metrics (Figure 4). Overall, the performance of the TM1 surrogate model is unlikely to be acceptable for a nacelle design process because it presents prediction errors above 10\% [20]. A slight refinement of the design space to build a meta-model with 650 samples (TM2) resulted in $\sigma_{L H S}$ of $9.4 \%, 7.8 \%$ and $8.9 \%$ for $C_{D-\text { cruise }}, \Delta C_{D-M a c h}$ and $C_{D-\text { spill }}$ with 


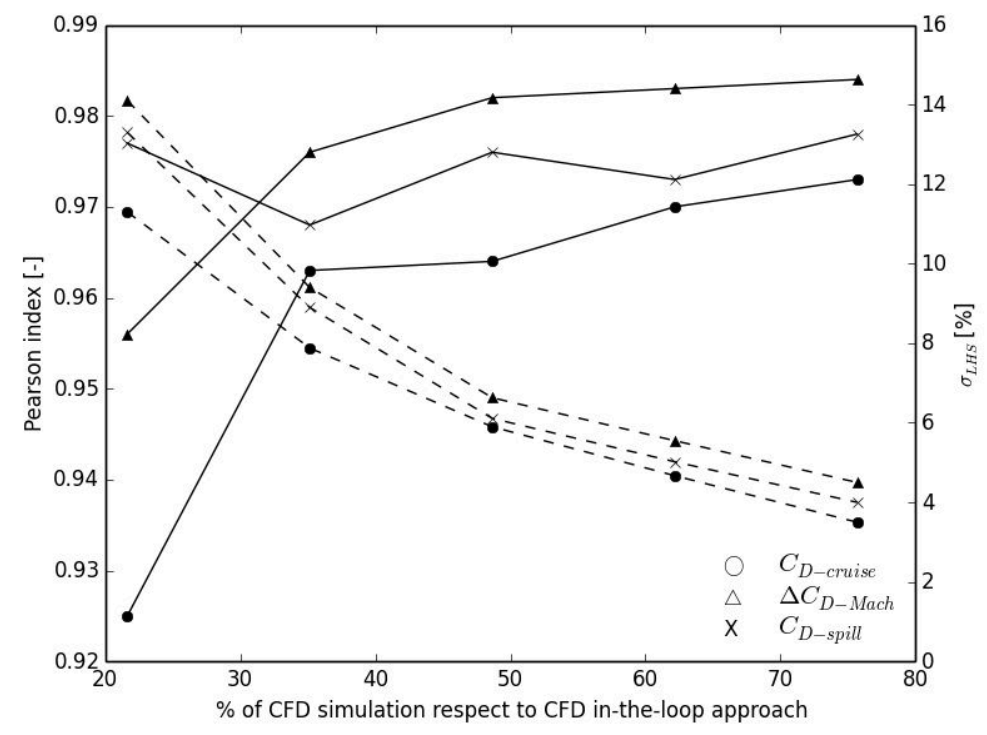

Figure 4: Linear correlation coefficient (solid line) and RMS error for the LOO cross-validation (dashed line) of the TM models

$N_{\text {Pearson }}>0.96$. As expected, the meta-model accuracy increases with the overall computational cost (Figure 4). For example, the TM5 model, which is built with a $75 \%$ computational cost with respect to the CFD in-the-loop method, has $\sigma_{L H S}$ below $5 \%$ for the three performance metrics of interest.

A key aspect during a multi-objective optimisation by means of RSMs is that the meta-model provides acceptable confidence in the predictive capability as well as drives the GA to the same set of optimum designs as the traditional CFD in-the-loop method. In this context, the five TM models built with the LHS DOEs (Table 1) were employed to drive the NSGA-II genetic algorithm, as described in Section 2.4, until the identification of five independent Pareto front surfaces. Figure 5 shows the Pareto front obtained with each TM model and the baseline from the CFD in-the-loop. Although the set of optimum nacelle designs form a Pareto front surface (e.g. Figure 3a), the comparison is only presented in the $C_{D-c r u i s e}-\Delta C_{D-M a c h}$ space which allows for an easier iden- 


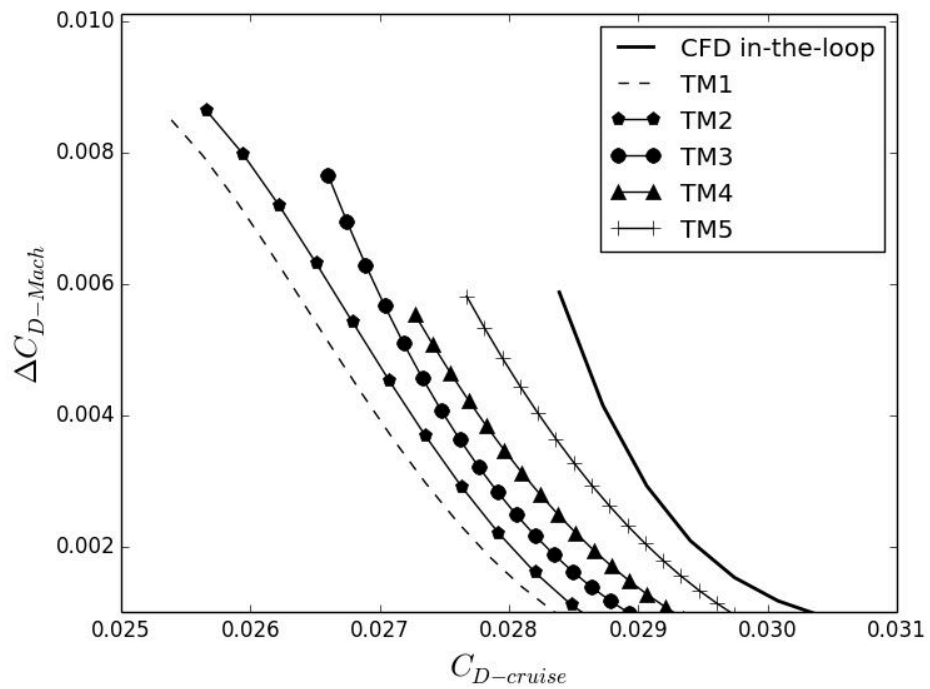

Figure 5: Pareto front comparison in the $C_{D-\text { cruise }}-\Delta C_{D-M a c h}$ space for the TM models and the baseline from the CFD in-the-loop method

tification of the projected Pareto front. This is also motivated by the fact that all non-dominated configurations present a $C_{D-\text { spill }}<0.1 \cdot C_{D-\text { cruise }}$, which is usually targeted during nacelle design. These results highlight the limitations of this MOO approach, based on a reduced-order model, to identify the same Pareto front as the traditional CFD in-the-loop method. To quantify the expected accuracy of the optimum nacelle designs, the Pareto optimal sets were calculated by direct CFD simulations and compared with the prediction of the meta-models. This allowed for a statistical computation of the accuracy of each meta-model in the region of the Pareto optimal design space $\left(\sigma_{L H S, P F}\right)$.

The structured RSM of the TM1 model identified a Pareto surface with an overall standard error in cruise drag $\left(C_{D-\text { cruise }}\right)$ of $\sigma_{L H S, P F}=9.03 \%$ (Figure 6 a), in which $25 \%$ of the optimum nacelle designs present an error in the $C_{D-\text { cruise }}$ prediction with respect to CFD above $10 \%$. In addition, the metamodel underpredicts the nacelle cruise drag for $98 \%$ of the optimal set, which is 
undesirable for a surrogate model in the early stage of the design process [20]. As the TM model is built with more nacelle design samples, the standard error of the Pareto front consistently decreases. For example, the TM4 and TM5 meta-models, which has associated a $62 \%$ and $75 \%$ CFD cost with respect to the CFD in-the-loop method, have $\sigma_{L H S, P F}=2.94 \%$ and $2.2 \%$, respectively (Figures $6 \mathrm{~d}$ and 6e). This confirms that the predictive accuracy of the structured RSMs can be improved by increasing the number of samples in the input data, but an additional key aspect of a MOO by meta-models is to ensure a similar convergence to a set of designs with respect to a higher fidelity method as the CFD in-the-loop.

In this respect, a more detailed investigation of the nacelle design with the lowest cruise drag identified with the TM1, TM2, TM3, TM4 and TM5 RSM models, referred as B1, B2, B3, B4 and B5 respectively, was carried out (Table 4). Compared to direct CFD simulations, the TM1 surrogate model underpredicts by $-17.7 \%$ the $C_{D-\text { cruise }}$ of the $\mathrm{B} 1$ nacelle design. This discrepancy is a direct implication of the overall quality of this meta-model (Figure 4). The B2 design, which is the minimum cruise drag found with the TM2 model, has a $-15.9 \%$ underprediction of $C_{D-\text { cruise }}$ with respect to CFD simulations. This highlights that acceptable surrogate models on the overall design domain can still present large prediction errors in the regions where optimum nacelle designs are concentrated (Figure 4). The other surrogate models, i.e. TM3, TM4 and TM5, found optimum nacelles designs at cruise conditions in which the difference with CFD simulations was below $10 \%$ (Table 4). The lift distribution along the B1 - B5 fan cowls have been compared to the sample A1 (Figure 7) to assess if the MOO by meta-models was capable of identifying the same nacelle design as the CFD in-the-loop method. While the A1 nacelle aero-line has a smooth, monotonic reduction in $M_{i s}$, the designs B1-B4 present a char- 
Table 4: Cross-validation of the optimum nacelle design in terms of $C_{D-\text { cruise }}$ for the different TM models

\begin{tabular}{lccc}
\hline & RSM prediction & CFD result & Difference [\%] \\
\hline Nacelle B1 & 0.0249 & 0.0302 & -17.7 \\
Nacelle B2 & 0.0251 & 0.0299 & -15.9 \\
Nacelle B3 & 0.0266 & 0.0284 & -6.2 \\
Nacelle B4 & 0.0273 & 0.0287 & -5.0 \\
Nacelle B5 & 0.0274 & 0.0286 & -4.0 \\
\hline
\end{tabular}

acteristically different flow topology with well-defined shocks. The sample B5, which has associated the largest CFD overhead cost, still presents differences with respect to the sample A1. It has a peak $M_{i s}$ of 1.37 and a deceleration of the flow that results in a shock-wave with pre-shock $M_{i s}$ of 1.10 . After the nacelle crown, both A1 and B5 aero-lines depict the same lift distribution.

Although it has been shown that the meta-model accuracy improves with the overall CFD overhead cost (Figure 4), the traditional method of surrogate modelling with a design space exploration was unable to drive the GA, at the sample rates considered in this work, to the same A1 nacelle design, that was obtained with the computationally expensive CFD in-the-loop approach (Figure 7). This highlights that new efficient MOO algorithms are required to overcome the limitations of this conventional strategy based on a design space exploration. The new proposed adaptive response surface modelling strategy is presented in Section 3.3 where its prediction capabilities as well as a comparison with the traditional methods are summarized.

\subsection{Nacelle Multi-Objective Optimisation by surrogate modelling based on an} adaptive response surface model

As it is envisaged that the evolutionary algorithm drives the optimiser to the regions were dominating individuals are located, a meta-model that contains CFD samples from different generations will improve the quality of the 


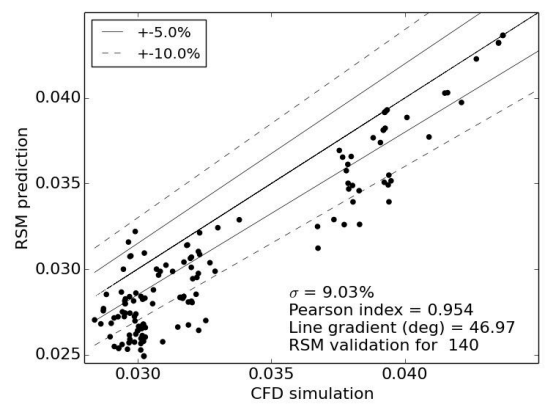

(a) Cross-validation TM1

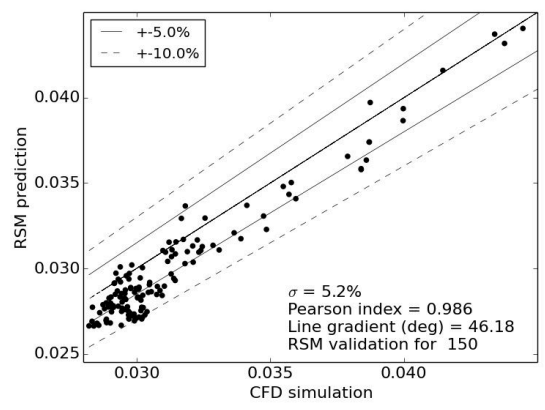

(c) Cross-validation TM3

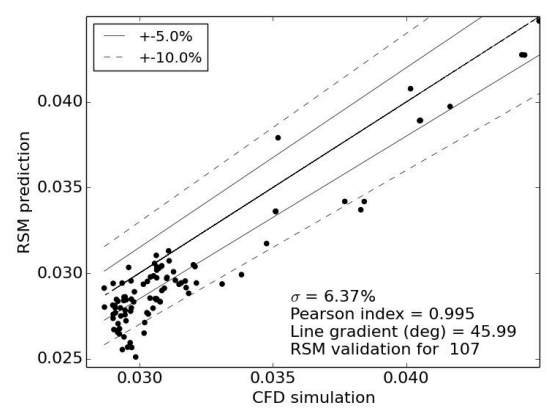

(b) Cross-validation TM2

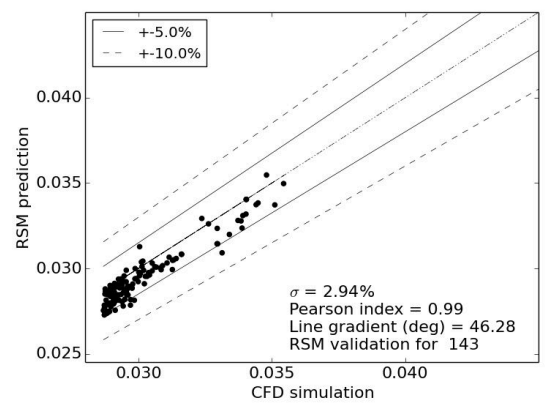

(d) Cross-validation TM4

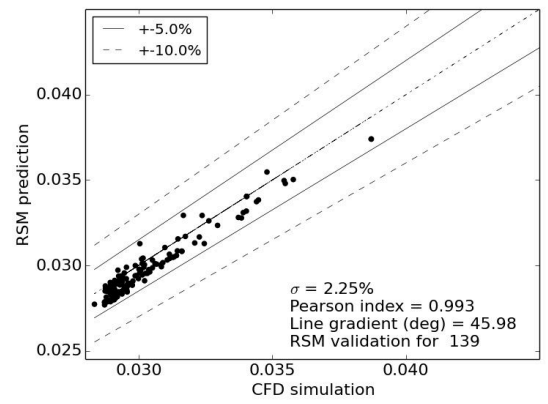

(e) Cross-validation TM5

Figure 6: Cross-validation for $C_{D-c r u i s e}$ of the Pareto front prediction obtained with the different TM models 


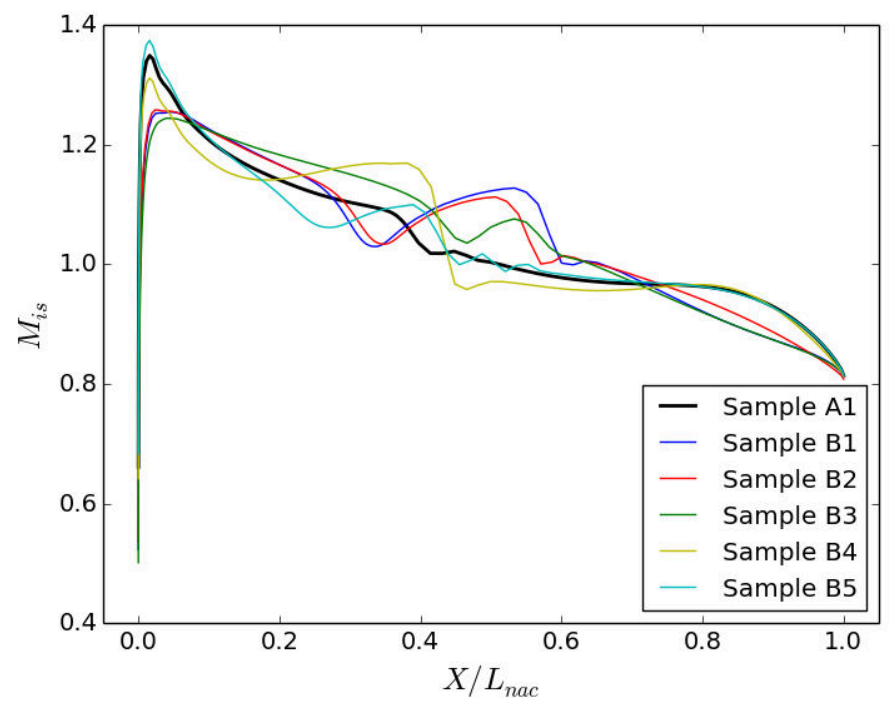

Figure 7: Lift distribution of optimum nacelle designs, in terms of $C_{D-c r u i s e}$, obtained with the different TM models

low order model in the regions of interest. While there are different methods that identify the regions of the design space with maximum predictions errors to encourage exploration, e.g. CV-Voronoi [48], this approach might not be suitable for surrogate-based nacelle optimisation. It may lead to extra CFD effort on parts on the design space that present large prediction uncertainties but low nacelle performance. As such, it is only required that the meta-model has an acceptable nacelle drag prediction in those non-optimal parts of the design space [20]. Nevertheless, methods to encourage exploration [48, 49] could be combined with the presented method to rapidly limit the feasible design space, which is the main difficulty for nacelle optimisation applications [22]. The proposed adaptive method is exploitative in nature and relies on the fact that the genetic algorithm is initially guided to the optimal parts of the design space. Within the context of this work, this is desirable as the GA was not found to be trapped in local optimal designs. This is demonstrated in the current work 
when the set of optimal designs obtained from this surrogate-based optimisation method is compared with the computationally expensive CFD in-the-loop approach. However, other applications might suffer this problem, which should be addressed by implementing mechanisms to encourage exploitation of the design space [50].

For the proposed adaptive method, four different surrogate models were created using the Gaussian Process Regression (GPR) based on a Kriging interpolation method as described in Section 3.2. A CFD in-the-loop optimisation with the well established method of a LHS containing 400 individuals and subsequent generations of 50 evaluations each was employed [23]. Then, the CFD data gathered until the $5^{\text {th }}, 10^{\text {th }}, 15^{\text {th }}$ and $20^{\text {th }}$ generation was used to build 4 different surrogate models, referred in the text as AM2, AM3, AM4 and AM5, respectively (Table 1). Subsequently, the meta-models were used to drive the GA to a set of Pareto optimal solutions.

For comparison purposes with the previous MOO method based on a design space exploration (Section 3.2), the computational cost to create each AM surrogate model is the same as to the equivalent TM (Table 1). Overall, both methods have similar values of the Pearson's index (Figures 4 and 8). The root mean square error of the nacelle cruise drag reduces in the AM models $\left(\sigma_{L H S+G A}\right)$. For example, while the TM2 presents a $\sigma_{L H S}=9.4 \%, 7.8 \%$ and $8.9 \%$ for $C_{D-\text { cruise }}, \Delta C_{D-M a c h}$ and $C_{D-\text { spill }}$, for the same computational overhead cost of the AM2 model, $\sigma_{L H S+G A}$ reduces slightly to $8.1 \% 7.2 \%$ and $7.7 \%$, respectively. The RMS error of the three performance metrics reduces with the total overhead cost (Figure 8). For instance, the AM5 meta-model, which has a $75 \%$ computational cost of the CFD in-the-loop method, has a prediction error on the overall design space of $\sigma_{L H S+G A}=1.8 \%, 1.6 \%$ and $2.0 \%$ for $C_{D-\text { cruise }}$, $\Delta C_{D-M a c h}$ and $C_{D-\text { spill }}$, respectively. This is an improvement of about a factor 


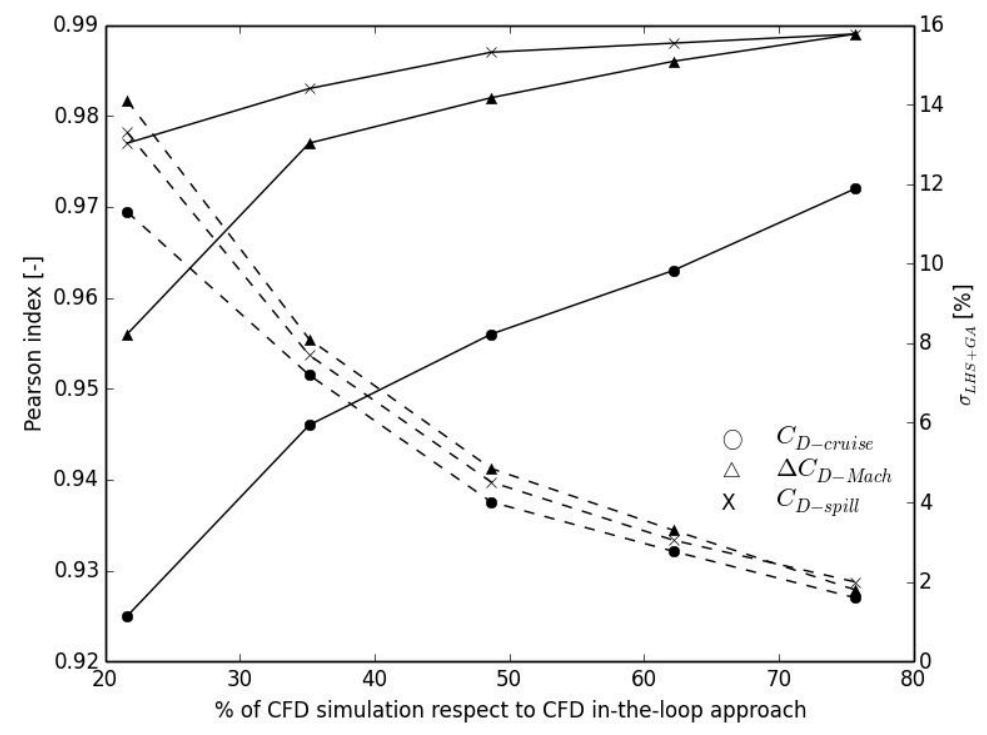

Figure 8: Linear correlation coefficient (solid line) and RMS error for the LOO cross-validation (dashed line) of the AM models

of two relative to the TM5 at the same computational cost.

Each meta-model built with this adaptive method, i.e. AM2-AM5, was used to drive the GA to a set of optimum nacelle aero-line designs. As previously, the projected Pareto front in the $C_{D-\text { cruise }}-\Delta C_{D-M a c h}$ space was compared against the baseline Pareto front from the CFD in-the-loop strategy (Figure 9). Compared to the Pareto fronts obtained with the TM models, at the same computational cost the accuracy on the identification of the set of optimum nacelle designs has been improved with the proposed AM approach. It is noted that the Pareto front from the AM5 meta-model underpredicts the cruise drag on the set of optimum nacelle designs by $0.5 \%$. Therefore, it is demonstrated that a set of similar nacelle designs can be obtained with a $25 \%$ reduction of the total computational overhead cost with respect to the CFD in-the-loop strategy.

A cross-validation with numerical simulations was carried out to quantify the accuracy of the AM meta-models in the Pareto front $\left(\sigma_{L H S+G A, P F}\right)$. The 


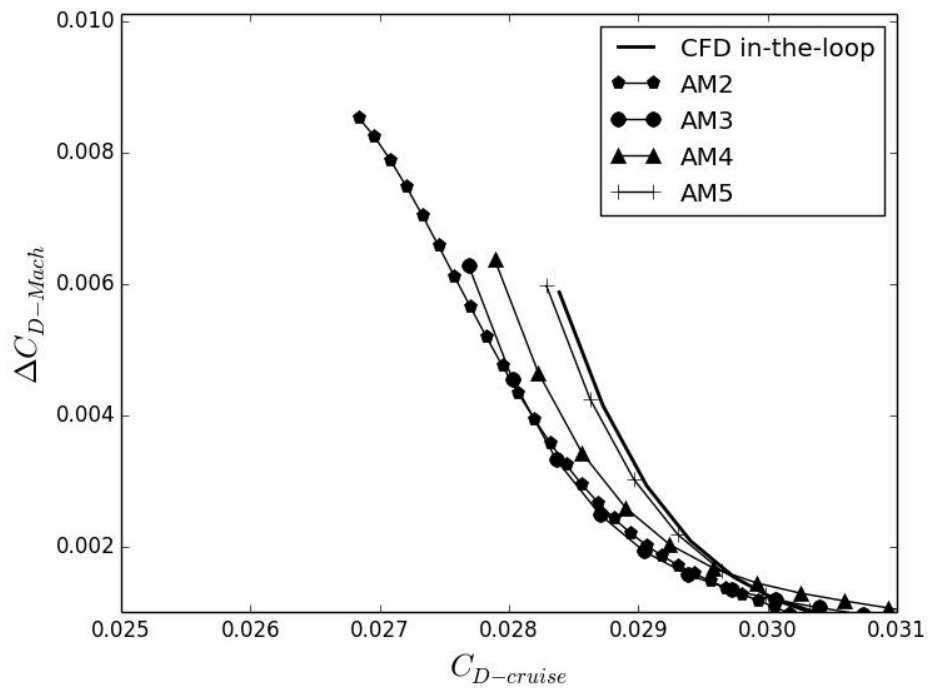

Figure 9: Pareto front comparison in the $C_{D-\text { cruise }}-\Delta C_{D-M a c h}$ space for the AM models and the baseline from the CFD in-the-loop method

AM2 model has a $\sigma_{L H S+G A, P F}=2.61 \%$, where less than $1 \%$ of the individuals have a prediction error above 10\% (Figure 10a). The AM3 structured RSM reduces the standard deviation on the Pareto front to $\sigma_{L H S+G A, P F}=1.73 \%$, where $98 \%$ of the nacelle design have a discrepancy on nacelle cruise drag below $5 \%$ (Figure 10b). As the surrogate model is built with more nacelle samples in the regions of interest, i.e. AM4 and AM5, the standard deviation $\sigma_{L H S+G A, P F}$ on the $C_{D \text {-cruise }}$ prediction reduces to $1.25 \%$ and $1.10 \%$, respectively (Figures 10c and 10d). These results highlight that for the same computational cost of the TM and AM methods, the proposed new strategy reduces by a factor of 2 the prediction error in the regions of the design space where the Pareto front is located. Thus, prediction-wise this new adaptive approach outperforms the widely employed surrogate model based on a design space exploration.

The nacelle designs with minimum cruise drag found with the AM2, AM3, AM4 and AM5 meta-models, and referred in the text as $\mathrm{C} 2, \mathrm{C} 3, \mathrm{C} 4$ and $\mathrm{C} 5$, 


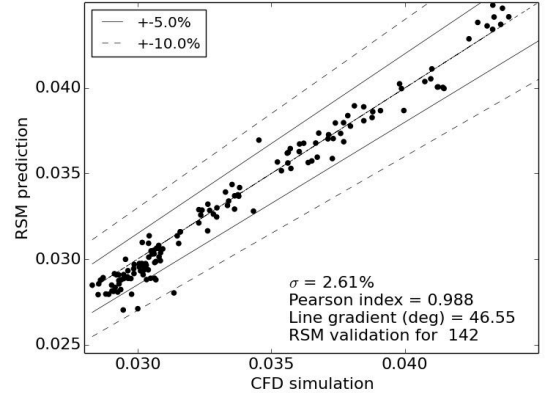

(a) Cross-validation AM2

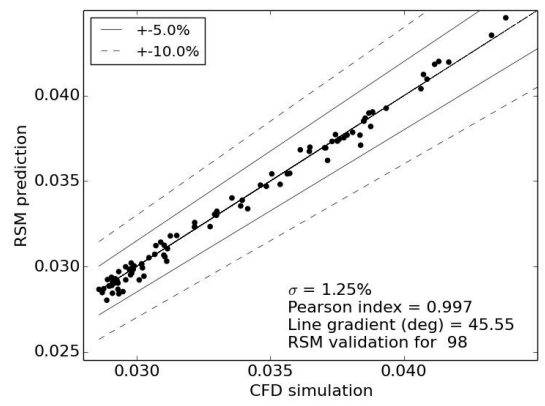

(c) Cross-validation AM4

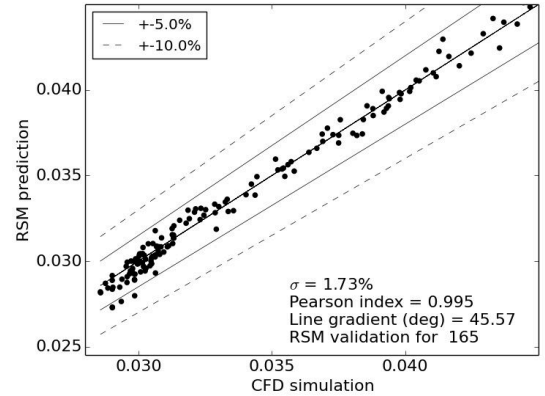

(b) Cross-validation AM3

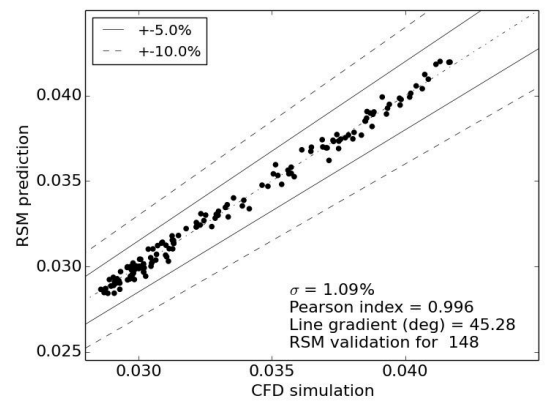

(d) Cross-validation AM5

Figure 10: Cross-validation for $C_{D \text {-cruise }}$ of the Pareto front prediction obtained with the different AM models 
have been further investigated. A cross-validation with CFD confirmed that the AM2 model found an aero-line design, $\mathrm{C} 2$, in which the $C_{D-\text { cruise }}$ was underpredicted by $-8.25 \%$ with respect to numerical simulations (Table 5 ). When the developed framework was driven by the AM3, AM4 and AM5 surrogate models, the GA found the $\mathrm{C} 3, \mathrm{C} 4, \mathrm{C} 5$ nacelle designs which had a nacelle drag underprediction of $-5.9 \%,-3.0 \%$ and $-0.7 \%$, respectively. This is a significant improvement compared to the traditional method based on a design space explication, i.e. TM model, where the underprediction of the nacelle designs B2-B5 obtained with the TM2-TM5 was $-15.9 \%,-6.2 \%,-5.0 \%$ and $-3.9 \%$ (Table 4 ). At this point, it is important to highlight that the $\mathrm{B}$ and $\mathrm{C}$ designs are the lowest $C_{D-\text { cruise }}$ predicted with their associated meta-models. Nevertheless, this does not guarantee that those designs are the configurations with minimum drag when evaluated with numerical simulations. As such, the lowest nacelle drag, based on CFD, was found for the B3 design (Table 5). During the cross-validation of the Pareto fronts presented in Figures 6 and 10, the minimum CFD $C_{D-\text { cruise }}$ of each surrogate-based optimisation was identified. Although the TM3, TM5 and AM2-AM5 meta-models found a nacelle design with minimum CFD $C_{D-\text { cruise }}=0.0283$, there was a large difference on the RSMs prediction uncertainty. For example, relative to the TM5 meta-model with a $2.5 \%$ discrepancy between RSM prediction and CFD, the AM5 model found a design with the same value of nacelle drag $\left(C_{D-\text { cruise }}=0.0283\right)$ with only a $0.1 \%$ discrepancy between RSM and CFD.

The lift distribution of the $\mathrm{C} 2-\mathrm{C} 5$ designs was compared to the reference A1 nacelle design found throughout the MOO based on the CFD in-the-loop strategy (Figure 12). Although the $\mathrm{C} 2-\mathrm{C} 4$ configurations has different flow topology than $\mathrm{A} 1$, the $\mathrm{C} 5$ aero-line has the same flow characteristics as A1 which confirms the capabilities of the proposed optimisation method to obtain the same nacelle 
Table 5: Cross-validation of the optimum nacelle design in terms of $C_{D-c r u i s e}$ for the different AM models (C2-C5 designs) and TM models (B1-B5 designs)

\begin{tabular}{cccccc}
\hline Model & $\begin{array}{c}\text { Cost relative } \\
\text { to CFD in-the-loop }\end{array}$ & Design & RSM prediction & CFD result & Difference [\%] \\
\hline AM2 & $35 \%$ & C2 & 0.0270 & 0.0294 & -8.3 \\
AM3 & $50 \%$ & C3 & 0.0273 & 0.0290 & -5.9 \\
AM4 & $62 \%$ & C4 & 0.0280 & 0.0289 & -3.0 \\
AM5 & $75 \%$ & C5 & 0.0284 & 0.0286 & -0.7 \\
\hline TM1 & $20 \%$ & B1 & 0.0249 & 0.0302 & -17.7 \\
TM2 & $35 \%$ & B2 & 0.0251 & 0.0299 & -15.9 \\
TM3 & $50 \%$ & B3 & 0.0266 & 0.0284 & -6.2 \\
TM4 & $62 \%$ & B4 & 0.0273 & 0.0287 & -5.0 \\
TM5 & $75 \%$ & B5 & 0.0274 & 0.0286 & -4.0 \\
\hline
\end{tabular}

design (Figure 12). It is acknowledged that genetic algorithms can converge rapidly to optimal solutions, and subsequent generations might contribute to only increase the Pareto front density. Within this work, the convergence of the $\mathrm{GA}$ was based on a reduction of the hypervolume below $1 \%$ on the last 3 generations, independently of how the GA is driven, i.e. CFD or surrogate model. It is important to highlight that despite the fast initial convergence that GAs usually provide, the benefits obtained with the AM method are not related to stopping the GA in a suboptimal set of solutions. Figure 11 demonstrates that due to the high non-linearity associated with the transonic flow of compact nacelles, the genetic algorithm requires several generations to identify optimal solutions within the final Pareto front. It compares the Pareto fronts in the $C_{D-\text { cruise }}-\Delta C_{D-\text { Mach }}$ space, for the last generations of the full CFD in-theloop approach (generation 30) and at the generations in which the surrogate models AM2, AM3, AM4 and AM5 were built, i.e. generations 5, 10, 15 and 20, respectively. As such, it confirms that the cost reduction found with the AM method appears from the utilization of a surrogate-based optimisation method.

A direct comparison of the statistical accuracy of the Pareto front obtained 


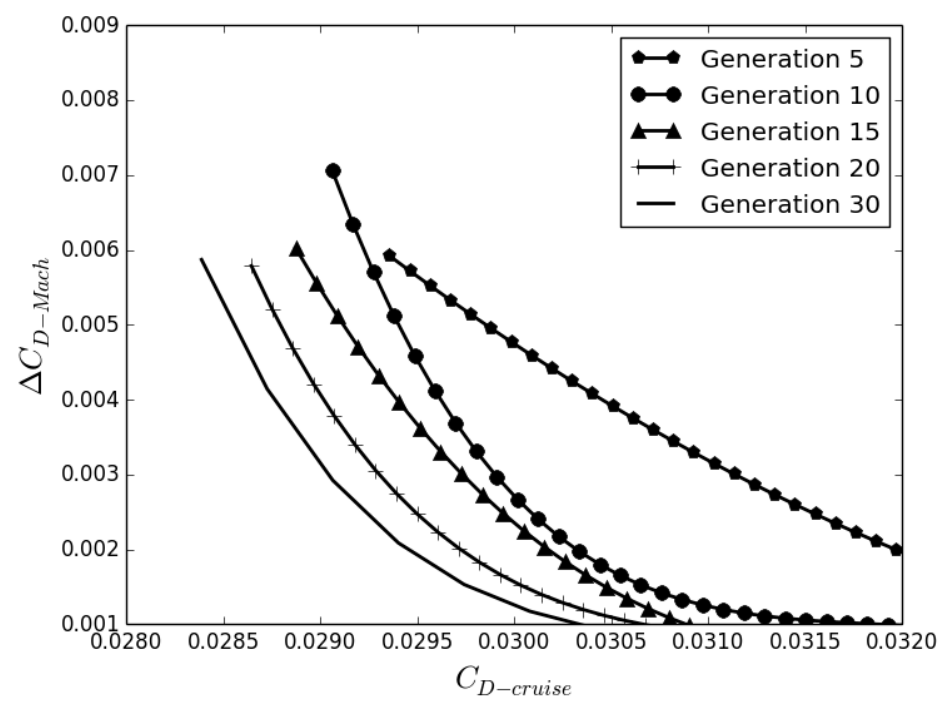

Figure 11: Pareto front comparison in the $C_{D \text {-cruise }}-\Delta C_{D-M a c h}$ for the generations 5,10 , 15,20 and 30

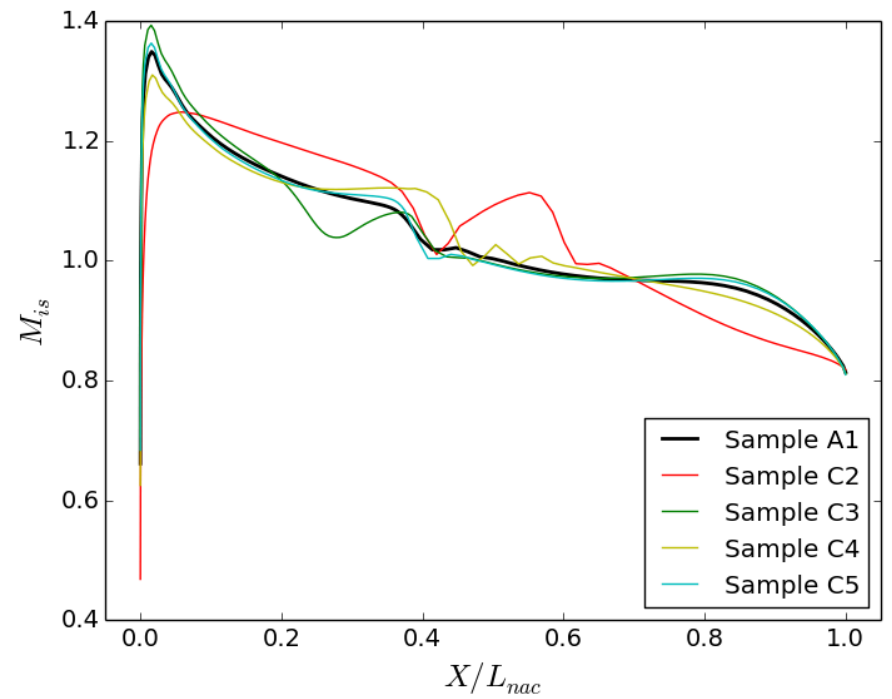

Figure 12: Lift distribution of optimum nacelle designs, in terms of $C_{D-c r u i s e}$, obtained with the different AM models 
with surrogate models from the design space exploration and from the adaptive method reveals the benefits on the prediction capabilities of the investigated algorithm (Figure 13). For early stage design it is typical to accept a threshold level of uncertainty in a trade-off with computational cost. Within aircraft design some methods consider overall uncertainty levels in the order of $5-10 \%$ as acceptable. Heidebrecht et al. [20] developed such a method for nacelle preliminary design purposes which provides rapid assessments of nacelle drag metrics as a function of flight Mach number, MFCR and typically eight geometric parameters for a $2 \mathrm{D}$ nacelle aeroline. The method uses a set of surrogate models based on an a priori CFD DSE to cover the eight-dimensional space. However, the method does not provide details of the nacelle aerodynamics or Mach number distributions. Within the nacelle design process, once the early preliminary design stage is complete, there is a need to increase the fidelity to enable more detailed assessments of the aerodynamics as well as mechanical loads. For example, for a $95 \%$ confidence level with a $2 \sigma$ of approximately $5 \%$, the conventional TM method provides a modest reduction of about $30 \%$ relative to the CFD-in-the-loop approach (Figure 13). However, the new adaptive RSM method provides a substantial improvement due to the accelerated convergence to the desirable parts of the design space. It is more than twice as efficient as the traditional surrogate modelling method and reduces the cost by about $65 \%$ relative to the CFD-in-the-loop approach (Figure 13).

The number of CFD generations required for the design space exploration is mainly related to the non-linearity of the problem, the degrees of freedom, the details of the GA parameters and the challenges to build accurate surrogate models. Consequently, it is difficult to know a priori the required number of generations to build a sufficiently accurate, yet computational efficient, surrogate model. Previous investigations based on a CFD in-the-loop method for 
single aero-lines required 30 generations of 50 individuals each with an initial design of experiments of 400 configurations [23, 22]. The thorough study carried out with the proposed adaptive method has highlighted that a set of Pareto optimal solutions with an acceptable prediction uncertainty at a preliminary stage of the design space can be obtained with only 5 CFD generations. On the other hand, it has been found that 20 generations were required to derive to a set of optimal solutions that were similar to the CFD in-the-loop approach based on a hypervolume criteria of a reduction lower than $1 \%$ in the last three generations.

Within the context of nacelle design from surrogate-based optimisation, it is usually preferred to overpredict the nacelle drag to establish a safety design margin. Nevertheless, the majority of optimal nacelle designs derived from the TM and AM models underpredict the cruise drag (Figures 6 and 10). Whilst the current method does not introduce a mechanism for conservative predictions [51], the thorough cross-validation with numerical simulations of the Pareto front presented in Figure 13 allows a statistical assessment of the uncertainty of optimal designs within the design space.

Although an evaluation of the drag characteristics is an important part of the low-order and adaptive MOO, it is also vital that the methods identify the same values of the geometric parameters as the higher fidelity approach. In this respect, it is useful to compare the geometric parameters for the identified Pareto optimal design sets. Figure 14 presents the box plot comparison of the three MOO methods considered, i.e. CFD in-the-loop, RSM from a design space exploration (TM5 model) and the proposed adaptive method (AM5 model), for the pertinent design variables of $f_{\max }, r_{\max } / r_{h i}$ and $f_{i f}$, which have a first order impact on the performance metrics [22]. Each box plots shows the median value (horizontal red line), the upper and lower quartiles (horizontal blue line) and the extreme values (horizontal black line), where the range has been normalised from 


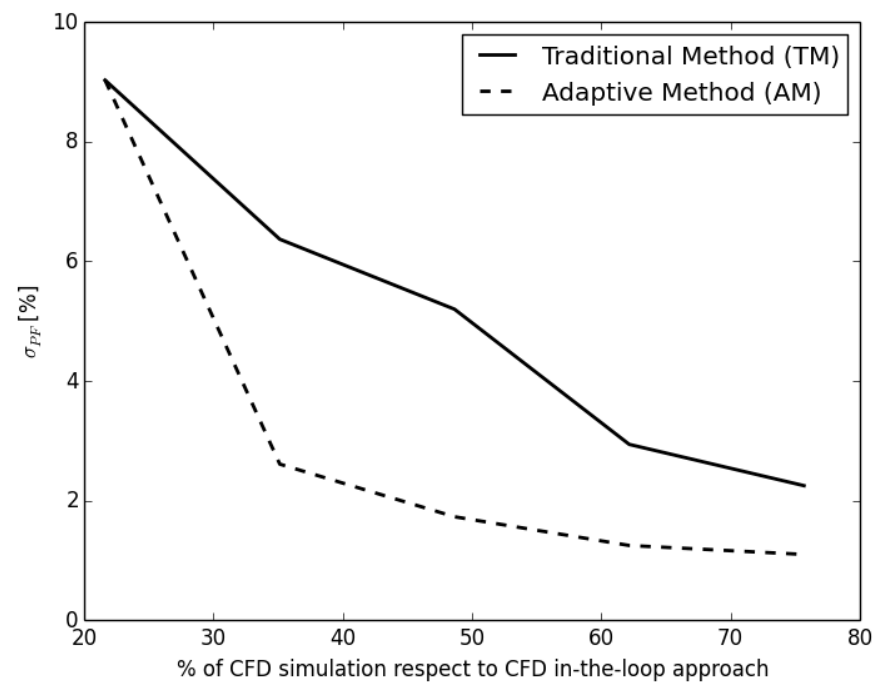

Figure 13: Prediction uncertainty of the surrogate models on the Pareto optimal set. Comparison of surrogate modelling by the traditional design space exploration and the proposed adaptive method

0 (lower design bound) to 1 (upper design bound). For example, while the CFD in-the-loop and the proposed adaptive method have similar normalised median value of $f_{\max }=0.63, r_{\max } / r_{h i}=0.61$ and $f_{i f}=0.40$ as well as quartiles and extreme values, the traditional MOO by surrogate modelling and DSE presents a $f_{\max }=0.48 . r_{\max } / r_{h i}=0.38, f_{i f}=0.13$ and significantly different quartiles and extreme values. This highlights that, with respect to the CFD in-theloop, a surrogate model built from the adaptive method drives the GA to the same regions of the design space. As such, this algorithm constitutes a useful approach for the multi-objective optimisation of non-linear problems by means of surrogate modelling and numerical simulations.

\section{Conclusions}

Within this investigation, a new adaptive surrogate modelling has been tested for the multi-objective optimisation of aero-engine nacelles. This re- 


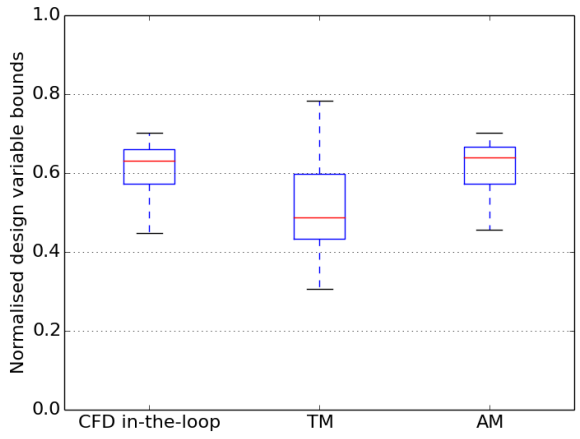

(a) $f_{\max }$

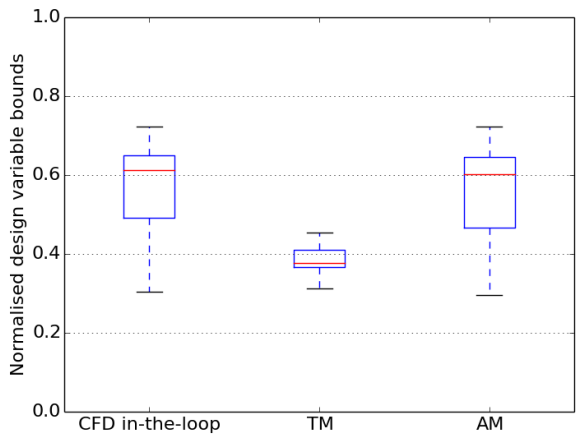

(b) $r_{\max } / r_{h i}$

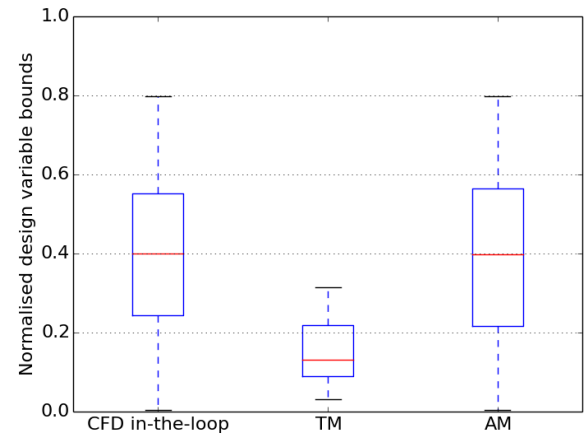

(c) $f_{i f}$

Figure 14: Box plots for the main pertinent design variables. Comparison of the three MOO strategies: CFD-in-the loop, surrogate modelling from a design exploration (TM5) and surrogate modelling with the proposed adaptive method (AM5) 
search has highlighted the difficulties to build accurate meta-models to predict non-linear transonic flow aerodynamics. As such, it was shown that a surrogate model based on a traditional design space exploration has large prediction errors in the regions of the design space where optimal geometries are located. The new adaptive surrogate model can obtain optimum nacelle geometries with a $25 \%$ reduction in computational cost with respect to the well-established CFD in-the-loop approach. Besides, the adaptive RSM strategy can determine a set of optimal designs with an average prediction error below $5 \%$, which is usually targeted during preliminary nacelle design, at a $65 \%$ reduction with respect to the standard CFD in-the-loop. Therefore, the developed method constitutes a useful strategy for the multi-objective optimisation of problems with transonic flow-fields, and computationally outperforms the current state of the art for nacelle design by surrogate modelling. 


\section{Acknowledgments}

The authors would like to thank the industrial partner, Rolls-Royce plc, for supporting this research.

\section{References}

[1] European Commission, Flightpath 2050: Europe's Vision for Aviation, Tech. Rep. ISBN 978-92-79-19724-6, Publications Office of the European Union (2011).

[2] F. Collier, R. Thomas, C. Burley, C. Nickol, C. Lee, M. Tong, Environmentally Responsible Aviation Project: Real Solutions for Environmental Challenges Facing Aviation, in: 27th International Congress of the Aeronautical Sciences, Paper No. 802, 2010.

[3] S. Skinner, H. Zare-Behtash, State-of-the-art in Aerodynamic Shape Optimisation Methods, Applied Soft Computing 62 (2018) 933-962.

[4] N. Birch, 2020 Vision: The Prospects for Large Civil Aircraft Propulsion, The Aeronautical Journal 104 (1038) (2000) 347-352.

[5] A. Guha, Optimum Fan Pressure Ratio for Bypass Engines with Separate or Mixed Exhaust Streams, Journal of Propulsion and Power 17 (5) (2011) $1117-1122$.

[6] D. Daggett, Ultra-Efficient Engine Technology Systems Integration and Environmental Assessment, Tech. Rep. CR-2002-211754, NASA (2002).

[7] W. Burgsmuller, S. Thome, Close Coupling of Very High-Bypass Engines in Combination with Take-Off/Landing, Notes on Numerical Fluid Mechanics 60 (1997) 95-102. 
[8] B. Rosenbaum, V. Schulz, Response Surface Methods for Efficient Aerodynamic Surrogate Models, N. Kroll, R. Radespiel, J. Burg and K. Sorensen (eds) Computational Flight Testing. Notes on Numerical Fluid Mechanics and Multidisciplinary Design 123 (2013) 113-129.

[9] N. E. Sevant, M. I. G. Bloor, M. J. Wilson, Aerodynamic Design of a Flying Wing Using Response Surface Methodology, Journal of Aircraft 37 (4) (2000) 562-569.

[10] E. Box, N. Draper, Empirical Model Building and Response Surfaces, New York: Wiley.

[11] D. Broomhead, D. Lowe, Radial Basis Functions, Multivariate Functional Interpolation and Adaptive Networks, Complex Systems 2 (1988) 321-355.

[12] T. Ray, W. Smith, Surrogate Assisted Evolutionary Algorithm for MultiObjective Optimization, in: 47th AIAA/ASME/ASCE/AHS/ASC Structures, Structural Dynamics and Materials Conference, AIAA Paper Number 2006-2050, 2006.

[13] G. Venturelli, E. Benini, Kriging-assisted Design Optimization of S-Shape supersonic compressor cascades, Aerospace Science and Technology 58 (2016) $275-297$.

[14] A. Hashimoto, S. Jeong, S. Obayashi, Aerodynamic Optimization of Nearfuture High-wing Aircraft, Transactions of the Japan Society for Aeronautical and Space Sciences 58 (2015) 73-82.

[15] I. Goulos, T. Stankowski, D. MacManus, P. Woodrow, C. Sheaf, Civil Turbofan Engine Exhaust Aerodynamics: Impact of Bypass Nozzle After-Body Design, Aerospace Science and Technology 73 (2018) 85-95. 
[16] W. Song, A. J. Keane, Surrogate-Based Aerodynamic Shape Optimization of a Civil Aircraft Engine Nacelle, AIAA Journal 45 (2007) 2565-2574.

[17] K. Deb, A. Pratap, S. Agarwal, T. Meyarivan, A Fast and Elitist Multiobjective Genetic Algorithm: NSGA-II, IEEE Transactions on Evolutionary Computation 6, (2) (2002) $182-197$.

[18] D. A. Masters, N. J. Taylor, T. C. S. Rendall, C. B. Allen, D. J. Poole, A Geometric Comparison of Aerofoil Shape Parameterization Methods, AIAA Journal 55 (2017) 1575-1589.

[19] S. Raghunathan, E. Benard, J. K. Watterson, R. K. Cooper, R. Curran, M. Price, H. Yao, R. Devine, B. Crawford, Key Aerodynamic Technologies for Aircraft Engine Nacelles, The Aeronautical Journal 110 (2006) 265-288.

[20] A. Heidebrecht, D. MacManus, Surrogate Model of Complex Non-Linear Data for Preliminary Nacelle Design, Aerospace Science and Technology 84 (2019) 399-411.

[21] D. Mitchell, Estimation of Spillage Drag for a Wide Range of Axisymmetric Intakes at M<1, Tech. Rep. ESDU-84004, ESDU (1984).

[22] F. Tejero, M. Robinson, D. G. MacManus, C. Sheaf, Multi-Objective Optimization of Short Nacelles for High Bypass Ratio Engines, Aerospace Science and Technology 91 (2019) 410-421.

[23] M. Robinson, D. MacManus, A. Heidebrecht, N. Grech, An Optimisation Method for Nacelle Design, in: 55th AIAA Aerospace Sciences Meeting, AiAA SciTech Forum, AIAA Paper Number 2017-0708, 2017.

[24] X. Fang, Y. Zhang, S. Li, , H. Chen, Transonic Nacelle Aerodynamic Optimization Based on Hybrid Genetic Algorithm, in: 17th AIAA/ISSMO Mul- 
tidisciplinary Analysis and Optimization Conference, AIAA AVIATION Forum, AIAA Paper Number 2016-3833, 2016.

[25] D. Sasaki, K. Nakahashi, Aerodynamic Optimization of an Over-the-WingNacelle-Mount Configuration, Hindawi Publishing Corporation Modelling and Simulation in Engineering 2011.

[26] Y. Zhong, S. Li, A 3D Shape Design and Optimization Method for Natural Laminar Flow Nacelle, in: Proceedings of ASME Turbo Expo 2017: Turbomachinery Technical Conference and Exposition, Paper Number GT201764379, Vol. 1, 2017, pp. 825-830.

[27] S. Li, Y. Zhong, A Turbofan-Engine Nacelle Shape Design and Optimization Method for Natural Laminar Flow Control, in: Proceedings of ASME Turbo Expo 2016: Turbomachinery Technical Conference and Exposition, Paper Number GT2016-57463, Vol. 1, 2016, pp. 825-830.

[28] B. Kulfan, Recent Extensions and Applications of the 'CST' Universal Parametric Geometry Representation Method, Aeronautical Journal 114 (1153) (2010) 157-176.

[29] R. Christie, A. Heidebrecht, D. G. MacManus, An Automated Approach to Nacelle Parameterisation Using Intuitive Class Shape Transformation Curves, Journal of Engineering for Gas Turbines and Power 139 (1153).

[30] Ansys Inc., 275 Technology Drive, Canonsburg, PA 15317, ANSYS ICEM CFD Tutorial Manual.

[31] Ansys Inc., 275 Technology Drive, Canonsburg, PA 15317, ANSYS FLUENT User's Guide.

[32] J. Enconniere, J. Ortiz-Carretero, V. Pachidis, Mission Optimisation for 
a Conceptual Coaxial Rotorcraft for Taxi Applications, Aerospace Science and Technology 72 (2018) 14-24.

[33] C. Alba, A. Elham, B. German, L. Veldhuis, A Surrogate-Based MultiDisciplinary Design Optimization Framework Modeling Wing-Propeller Interaction, Aerospace Science and Technology 78 (2018) 721-733.

[34] J. C. Helton, F. J. Davis, Latin Hypercube Sampling and the Propagation of Uncertainty in Analyses of Complex Systems, Reliability Engineering \& System Safety 81 (2003) 23-69.

[35] M. D. McKay, R. J. Beckman, W. J. Conover, A Comparison of Three methods for Selecting Values of Input Variables in the Analysis of Output from a Computer Code, Technometrics 21 (2) (1979) 239-245.

[36] F. R. Menter, Two-Equation Eddy-Viscosity Turbulence Models for Engineering Applications, AIAA Journal 32 (8) (1994) 1598-1605.

[37] M. Robinson, D. MacManus, C. Sheaf, Aspects of Aero-Engine Nacelle Drag, in: Proceedings of the Institution of Mechanical Engineers, Part G: Journal of Aerospace Engineering, 2018.

[38] P. Roache, A Method for Uniform Reporting of Grid Refinement Studies, Journal of Fluids Engineering 116 (3) (1994) 405-413.

[39] M. Langley, The Design of Axisymmetric Cowls for Podded Nacelles for High Bypass Ratio Turbofan Engines, Tech. Rep. 3846, Aeronautical Research Council (1979).

[40] T. Simpson, T. M. Mauery, J. J. Korte, F. Mistree, Kriging Models for Global Approximation in Simulation-Based Multidisciplinary Design Optimization, AIAA Journal 39 (12) (2001) 2233-2241. 
[41] R. Kohavi, A Study of Cross-Validation and Bootstrap for Accuracy Estimation and Model Selection, in: Proceedings of the Fourteenth International Joint Conference on Artificial Intelligence, Vol. 2, 1995, pp. 11371143.

[42] M. van Oijen, Bayesian Methods for Quantifying and Reducing Uncertainty and Error in Forest Models, Current Forestry Reports 3 (2017) 269-280.

[43] H. Hotelling, New Light on the Correlation Coefficient and its Transforms, Journal of the Royal Statistical Society 15 (2) (1953) 193-232.

[44] K. Deb, Multi-Objective Optimisation Using Evolutionary Algorithms, Tech. Rep. 3846, Wiley, Chichester, UK (2013).

[45] M. Robinson, The Aerodynamics of Aero-Engine Nacelles, Tech. rep., PhD thesis, Cranfield University (2018).

[46] L. J. Eshelman, J. Schaffer, Real-Coded Genetic Algorithms and IntervalSchemata, Foundations of Genetics Algorithms 2 (1993) 187-202.

[47] E. Zitzler, L. Thiele, Multiobjective Evolutionary Algorithms: A Comparative Case Study and the Strength Pareto Approach, IEEE Transactions on Evolutionary Computation 3 (1999) 257-271.

[48] H. Liu, S. Xu, X. Wang, S. Yang, J. Meng, A Multi-Response Adaptive Sampling Approach for Global Metamodeling, Proceedings of the Institution of Mechanical Engineers, Part C: Journal of Mechanical Engineering Science 232 (1) (2018) 3-16.

[49] V. Aute, K. Saleh, O. Abdelaziz, S. Azarm, R. Radermacher, CrossValidation Based Single Response Adaptive Design of Experiments for Kriging Metamodeling of Deterministic Computer Simulations, Structural and Multidisciplinary Optimization 48 (2013) 581-605. 
[50] K. Crombecq, D. Gorissen, D. Deschrijver, T. Dhaene, A Novel Hybrid Sequential Design Strategy for Global Surrogate Modeling of Computer Experiments, SIAM Journal on Scientific Computing 33 (4) (2011) 19481974.

[51] V. Picheny, N. Kim, R. T. Haftka, N. V. Queipo, Conservative Predictions Using Surrogate Modeling, in: 49th AIAA/ASME/ASCE/AHS/ASC Structures, Structural Dynamics, and Materials, AIAA Paper Number 2018-1716, 2008. 
2019-05-29

Surrogate-based aerodynamic optimisation of compact nacelle aero-engines

Tejero, Fernando

Elsevier

Tejero F, MacManus DG, Sheaf C. (2019) Surrogate-based aerodynamic optimisation of

compact nacelle aero-engines. Aerospace Science and Technology, Volume 93, October 2019,

Article number 105207

https://doi.org/10.1016/j.ast.2019.05.059

Downloaded from Cranfield Library Services E-Repository 